\title{
Hearing the Zero Locus of a Magnetic Field
}

\author{
Richard Montgomery \\ Department of Mathematics, University of California, Santa Cruz, CA 95064, USA. \\ E-Mail: rmont@cats.ucsc.edu
}

Received: 31 March 1994/in revised form: 6 July 1994

\begin{abstract}
We investigate the ground state of a two-dimensional quantum particle in a magnetic field where the field vanishes nondegenerately along a closed curve. We show that the ground state concentrates on this curve as $e / h$ tends to infinity, where $e$ is the charge, and that the ground state energy grows like $(e / h)^{2 / 3}$. These statements are true for any energy level, the level being fixed as the charge tends to infinity. If the magnitude of the gradient of the magnetic field is a constant $b_{0}$ along its zero locus, then we get the precise asymptotics $(e / h)^{2 / 3}\left(\mathrm{~b}_{0}\right)^{2 / 3} E_{*}+O(1)$ for every energy level. The constant $E_{*} \simeq .5698$ is the infimum of the ground state energies $E(\beta)$ of the anharmonic oscillator family $-\frac{d^{2}}{d y^{2}}+\left(\frac{1}{2} y^{2}-\beta\right)^{2}$.
\end{abstract}

\section{Introduction}

We investigate the asymptotics of a two-dimensional quantum particle in a magnetic field as $\lambda=e / h$ tends to infinity. Here $e$ is its charge and $h$ is Planck's constant. The Hamiltonian of Schrodinger's equation is the covariant Laplacian associated to a connection whose curvature is the magnetic field. Geometrically speaking, the parameter $\lambda$ is the Chern number, or power, of the line bundle on whose sections this Laplacian acts. If the magnetic field is bounded away from zero by some constant $B_{0}$, then it is well-known that the particle's ground state energy $E_{1}(\lambda)$ satisfies

$$
E_{1}(\lambda) \geqq\left|\lambda B_{0}\right|
$$

We are interested in the case where the field does vanish. We will assume that it vanishes along a closed curve $C$ and that its gradient there is nonzero. Our main result is that in this case the ground state concentrates along $C$ as $\lambda \rightarrow \infty$ and that its energy satisfies:

$$
E_{1}(\lambda)=. O\left(\lambda^{2 / 3}\right)
$$

More generally, for every eigenvalue below the continuous spectrum, the same energy bounds and eigensection concentration holds. 
If the gradient of the magnetic field has constant nonzero magnitude $b_{0}$ along $C$ then we can be more precise about the spectral asymptotics. For each fixed $j$ the $j^{\text {th }}$ eigenvalue satisfies

$$
E_{j}(\lambda)=\lambda^{2 / 3} b_{0}^{2 / 3} E_{*}+o\left(\lambda^{2 / 3}\right)
$$

where the positive constant $E_{*}$ is defined to be the infimum of the ground state energies $E(\beta)$ of the anharmonic oscillator family $-\frac{d^{2}}{d y^{2}}+\left(\frac{1}{2} y^{2}-\beta\right)^{2}$. With the help of Cesar Castilho we have $E_{*} \simeq .5698$.

There is a fair amount of work regarding the spectrum of covariant Laplacians. Most authors assume that the magnetic field is nowhere vanishing, or in higher dimensions, that the curvature is fat, which means as nondegenerate as possible. In $[12,13$, and 14] Guillemin and Uribe relate the spectral asymptotics to the classical Hamiltonian flows under the assumption of fatness. In [7] and [8] Demailly weakens the assumption of pointwise nondegeneracy of the curvature and uses his resulting asymptotics to answer a conjecture in complex complex differential geometry. The papers of Simon and co-authors [27,6] and references therein, contain a host of varied results concerning the spectrum for magnetic fields on the plane.

The motivation for this work was our discovery of a peculiar classical phenomenon. The classical motions $\gamma(t)=(x(t), y(t))$ of a particle in a magnetic field satisfy the following constrained variational principle: minimise the arclength between two given endpoints on the surface, subject to the constraint that the "flux" $\Phi=\int_{\gamma} A$ between these two points is fixed. $A$ is the one-form potential for the magnetic field $B: d A=B \times$ (area form). In [25] we showed that, in addition to the classical motions, every sufficiently short arc of the zero locus $C$ of the magnetic field is also a solution to this minimization problem. Such arcs are critical points of $\Phi$. In some sense, the extraneous solution $C$ corresponds to a classical motion for which the particle's charge is infinite. Our original question was "Does the extraneous solution persist upon quantization?" We find that not only does it persist, but it is the dominant feature as $\lambda=e / h$ tends to infinity.

1.1. Sublaplacians and Nilpotent Groups. The correspondence between the spectral asymptotics of Laplacians and Riemannian geodesics is well-known. We believe the phenomenon described here is one of the first instances of a correspondence between the asymptotics of subLaplacians and singular geodesics for their corresponding SubRiemannian geometries. We will define these terms momentarily.

Suppose that the underlying two-dimensional space for our magnetic field problem is flat with metric $d x^{2}+d y^{2}$. Then the family of Hamiltonians which we investigate is

$$
H(\lambda)=-\left(\frac{\partial}{\partial x}-i \lambda A_{x}(x, y)\right)^{2}-\left(\frac{\partial}{\partial y}-i \lambda A_{y}(x, y)\right)^{2}
$$

where $A_{x} d x+A_{y} d y$ represents the connection form, or vector potential for the field. Introduce a circular variable $z$ and consider the hypoelliptic operator

$$
H=-\left(\frac{\partial}{\partial x}-A_{x} \frac{\partial}{\partial z}\right)^{2}-\left(\frac{\partial}{\partial y}-A_{y} \frac{\partial}{\partial z}\right)^{2}
$$


Decomposing wave functions into Fourier modes relative to $z$ we find:

$$
H=\bigoplus_{\lambda \in \mathbb{Z}} H(\lambda)
$$

The operator $H$ is an example of a subLaplacian which is hypoelliptic according to a theorem of Hormander: By a subLaplacian we mean a second order linear differential operator of the form $H=\sum_{j=1}^{k} X_{j}^{2}+X_{0}$, where the $X_{j}$ are smooth vector fields on a n-manifold $Q, n>k . H$ is called hypoelliptic if whenever $u$ is a distributional solution to the equation $H u=f$ with $f$ smooth then $u$ is also smooth. Hormander's theorem [19] asserts that a subLaplacian is hypoelliptic provided the $X_{j}$ are Lie bracket-generating, which is to say that the $X_{j}$ together with their iterated Lie brackets $\left[X_{i}, X_{j}\right],\left[X_{l},\left[X_{j}, X_{k}\right]\right], \cdots$ span the entire tangent space $T Q$. The $\mathrm{H}$ for our magnetic problem is the hypoelliptic. It takes two brackets to generate along the zero locus of B and one everywhere else.

The differential geometry underlying subLaplacians is called subRiemannian geometry [28] or Carnot-Caratheodory geometry [11]. It consists of a rank $k$ distribution $\mathscr{D} \subset \mathrm{T} Q$ together with a fiber inner product on $\mathscr{D} . \mathscr{D}$ is the span of the $X_{j}$ which we declare to be orthonormal, thus defining the inner product. In our magnetic field problems $Q$ is a 3-manifold, the total space of a circle bundle with connection $A$, over a two-dimensional Riemannian surface $M^{2}$. The 2-plane field $\mathscr{D} \subset \mathrm{T} Q$ is the horizontal distribution $\left\{d z-A_{x} d x-A_{y} d y=0\right\}$ associated to the connection A and the vector fields $X_{1}, X_{2}$ are the horizontal lifts of an orthonormal frame for $M$. The operator $\mathrm{H}$ is the so-called covariant or horizontal Laplacian. It commutes with the circle action on $Q$ so we can decompose it according to Fourier modes (representations of the circle),

$$
\begin{aligned}
H & =\bigoplus_{\lambda \in \mathbb{Z}} H(\lambda), \\
L^{2}(Q) & =\bigoplus_{\lambda \in \mathbb{Z}} \Gamma(\lambda),
\end{aligned}
$$

where $\psi \in \Gamma(\lambda)$ if $\psi\left(e^{i \theta} q\right)=e^{i \lambda \theta} \psi(q)$, where $q \mapsto e^{\imath \theta} q$ denotes the circle action. The spaces $\Gamma(\lambda)$ are naturally identified with the $\lambda^{\text {th }}$ tensor product of the Hermitian line bundle $L$ for which $Q$ is the space of unit vectors in its dual $L^{*}=$ $L^{-1}$. We are interested in the asymptotics as $\lambda \rightarrow \infty$. In the general case $\lambda$ will be some measure of the components transverse to $\mathscr{D}$ of the differentials of functions.

Let $\Omega_{\mathscr{D}}$ be the set of absolutely continuous paths in $Q$ whose derivatives lie in $\mathscr{D}$ when they exist. The length of such a path $\gamma:[0, T] \rightarrow Q$ is defined as the integral $\sqrt{\langle\gamma}, \dot{\gamma}\rangle d t$. We use this length functional to define minimizing subRiemannian geodesics, geodesics, and a distance function as in Riemannian geometry. Let $h: T^{*} Q \rightarrow \mathbb{R}$ be the principal symbol for our subLaplacian. Thus

$$
h=\frac{1}{2} \sum_{j=1}^{k}\left(P_{j}\right)^{2}
$$

where $P_{J}(q, p)=p\left(X_{j}(q)\right)$ for $p \in T_{q}^{*} Q$. (So $\mathrm{H}$ is a quantization of h.) The projections $\gamma \subset Q$ to $Q$ of the solutions $\xi \subset T^{*} Q$ to Hamilton's equations for $\mathrm{h}$ are locally-in-time minimizing geodesics. For a while it was thought that these accounted for all geodesics. However, the peculiar classical phenomenon just referred 
to provides a counterexample: the horizontal lifts to $Q$ of the zero locus $C \subset M^{2}$ in our magnetic problem are locally minimizing geodesics which are not projections of solutions to Hamilton's equations.

These curves are the first examples of strictly singular geodesics. An integral curve for a distribution $\mathscr{D}$ is called singular if it is a critical point for the endpoint map end: $\Omega_{\mathscr{D}} \rightarrow Q \times Q$ which sends a path $\gamma$ to its endpoints end $(\gamma)=(\gamma(0), \gamma(T))$. Since our work Liu and Sussmann [22] have found a plethora of singular minimizers for rank 2 distributions in $n$-dimensions, of which ours is the simplest case. The curves which they investigate are the same class investigated by Bryant and Hsu [3] who showed that they are isolated points in the $C^{1}$-topology on $\Omega_{\mathscr{D}} \cap$ end $^{-1}\left(q_{0}, q_{1}\right)$ mod reparameterisations.

There are hints (Sussmann, private communication; Bismut [1]) that the smalltime asymptotics for the subLaplacian's heat kernel is dominated by contributions from singular curves. There is also evidence for the conjecture that the presence of rigid singular geodesics signals the breakdown of a property called analytic hypoellipticity for the subLaplacian. (An operator is called analytic hypoelliptic if $H u=f$ with $f$ analytic implies that $u$ is analytic.) A stronger conjecture was made by Treves [29] for the case of general pseudodifferential operators with nonnegative symbol $h$. In this generality, singular curves correspond to the projections of nonvanishing Cartan characteristics for the restriction of the canonical two-form $\omega$ to the characteristic set $\{h=0\} \subset T^{*} Q .(\xi:[0, T] \rightarrow\{h=0\}$ is a Cartan characteristic if $\omega(\dot{\xi}, \cdot)=0$ ). Evidence supporting this conjecture is provided by work of Helffer on invariant subLaplacians on nilpotent groups. We describe Helffer's work briefly. It is relevant to our work in more ways than one.

The subRiemannian analogue of the Riemannian tangent space at $q$ is called the nilpotentization of $(\mathscr{D}, Q)$ at $q$ and is an $n$-dimensional graded nilpotent Lie group $G=G_{q}$ with a rank $k$ subRiemannian structure. (See $[26,22,24]$.) For this and other reasons invariant subLaplacians on such groups have been the subject of much study. (In addition to some of the above references, see also [16,17], and references therein.) Helffer analyses such subLaplacians in terms of their spectral decomposition $\oplus_{\lambda} H(\lambda)$, where now $\lambda$ runs over $G_{1}$ the space of irreducible representations of $G$. He proves (Theorem 1.3, [15]) that the subLaplacians arising from the nilpotentization of the rank 2 examples of Bryant-Hsu-Liu-Sussmann fail to be analytic hypoelliptic. The simplest rank 2 nilpotentization is the group whose Lie algebra is the four-dimensional Engel algebra. This has basis $\{X, Y, W, Z\}$ and only non-vanishing brackets $[Y, X]=W,[Y, W]=Z$. Its subLaplacian is $X^{2}+Y^{2}$. Helffer calculates that for a certain family of representations $\lambda$ the corresponding covariant Laplacians $H(\lambda)$ are precisely the anharmonic family of oscillators $-\left(\frac{\partial}{\partial y}\right)^{2}+\left(\beta+\frac{1}{2} b y^{2}\right)^{2}$ which are the key to our spectral analysis. They are also the key to Helffer's Theorem 1.3.

This coincidence is perhaps not so surprising. A local normal form for distributions of the type we investigate (generic degeneracy of a contact distribution) near the zero locus was found by Martinet [23] and asserts that the distribution can be framed by vector fields $Y=\frac{\partial}{\partial y}$ and $X=\frac{\partial}{\partial x}+\frac{1}{2} y^{2} \frac{\partial}{\partial z}$. (These will typically not be orthonormal.) The Engel algebra can be realised as the Lie algebra generated by the vector fields $\bar{Y}=\frac{\partial}{\partial y}$ and $\bar{X}=\frac{\partial}{\partial x}+y \frac{\partial}{\partial w}+\frac{1}{2} y^{2} \frac{\partial}{\partial z}$ on $\mathbb{R}^{4}$. Observe that $\pi_{*} \bar{X}=X, \pi_{*} \bar{Y}=Y$, where $\pi$ is the projection $\pi(x, y, z, w)=(x, y, z)$. So the Engel subLaplacian projects to an approximation of our $H$, the approximation which 
we use to develop our asymptotics. This approximation is exact when the scalar magnetic field is $B=y$ on the flat plane.

\subsection{Physical Considerations.}

$$
H=-\frac{\hbar^{2}}{2 m}\left\{\left(\frac{\partial}{\partial x}-i \frac{e}{h} A_{x}(x, y)\right)^{2}+\left(\frac{\partial}{\partial y}-i \frac{e}{h} A_{y}(x, y)\right)^{2}\right\}
$$

is the Hamiltonian for a spinless nonrelativistic planar particle of charge $e$ and mass $m$ in a magnetic field perpendicular to the plane whose signed magnitude is $B$. Here $A=A_{x} d x+A_{y} d y$ is the vector potential for $B$ so that $B=\frac{\partial}{\partial y} A_{x}-\frac{\partial}{\partial x} A_{y}$, or $B d x \wedge d y=d A$. If the magnetic field is a constant $B=B_{0}$ then the ground state energy is

$$
E_{1}=\frac{1}{2} \frac{\hbar^{2}}{m} \frac{\left|e B_{0}\right|}{\hbar}
$$

The bound (1) above states that if $B$ is not constant but instead satisfies a bound $B>B_{0}$ (or is less than $-B_{0}$ ) then this energy is a lower bound for the true energy.

Our result concerning the case where the field vanishes along $C$ is that

$$
E_{j}(e) \sim \frac{\hbar^{2}}{m}\left\{\left(\frac{\left|e b_{0}\right|}{\hbar}\right)^{2 / 3} E_{*}+o(1)\right\}
$$

in case the magnitude $b_{0}$ of $\nabla B$ is constant along $C$. The scale at which this asymptotics is valid is

$$
\frac{e b_{0}}{h} \gg \frac{1}{\ell^{3}},
$$

where $\ell$ encodes the length scales associated to $C$, for example, its length, the inverse of its minimum curvature, and $\frac{b_{0}}{\left|\nabla^{2} B\right|}$.

The operator which we will study is obtained from the above Hamiltonian by eliminating units and multiplying by 2 . Thus replace $\frac{\hbar^{2}}{m}$ by 1 , set $e / \hbar=\lambda$, and multiply by 2 to obtain

$$
H(\lambda)=-\left(\frac{\partial}{\partial x}-i \lambda A_{x}(x, y)\right)^{2}-\left(\frac{\partial}{\partial y}-i \lambda A_{y}(x, y)\right)^{2} .
$$

\section{Formulation and Results}

We will formulate our results on curved surfaces. So, let $M$ be an oriented complete Riemannian surface, possibly with boundary. A magnetic field on $M$ is the curvature two-form of a connection ( $U(1)$-gauge field) $A$ for a complex Hermitian line bundle $\pi: L \rightarrow M$ over $M$. The magnetic field can be represented by a scalar field $B$ on $M$ according to the rule

$$
d A=\pi^{*} B d^{2} x
$$

where $d^{2} x$ represents the area form on $M$.

The quantum Hamiltonian $H=H(A)$ for our particle is the covariant Laplacian:

$$
H=D_{A}^{*} D_{A} \text {. }
$$


In local coordinates $x^{1}, x^{2}$ on $M$ :

$$
H \psi=-\frac{1}{\sqrt{g}} \Sigma D_{\mu}\left(g^{\mu v} \sqrt{g} D_{v} \psi\right) .
$$

Here $g^{\mu v}$ is the inverse metric on $M, \sqrt{g} d x^{1} d x^{2}=d^{2} x$ is the area form,

$$
D_{\mu}=\frac{\partial}{\partial x^{\mu}}-i A_{\mu}
$$

is the covariant derivative in the $x^{\mu}$ direction, and $A=A_{1} d x^{1}+A_{2} d x^{2}$ is the connection one-form relative to the local trivialization in which the section $\psi$ is represented,

$$
D_{A} \psi=\left(D_{1} \psi\right) d x^{1}+\left(D_{2} \psi\right) d x^{2}
$$

is a section of $T^{*} M \otimes L$. The operator $D_{A}^{*}$ is the adjoint of $D_{A}$ relative to the $L^{2}$ pairing for sections of $L$ and of $T^{*} M \otimes L$. If $M$ has boundary we impose Dirichlet boundary conditions: $\phi=0$ on the boundary of $M$. If $M$ is noncompact we insist that the sections are square integrable.

The particle couples to the magnetic field through its charge $\lambda$ and the minimal coupling rule:

$$
\frac{\partial}{\partial x^{\mu}} \rightarrow D_{\mu}(\lambda)=\frac{\partial}{\partial x^{\mu}}-i \lambda A_{\mu} .
$$

Geometrically, minimal coupling is achieved by replacing the line bundle $L$ by its $\lambda$-fold tensor product:

$$
L^{\lambda}=L \otimes L \otimes \ldots \otimes L(\lambda \text { times }) .
$$

(Negative $\lambda$ corresponds to powers of the dual of $L$.) For if $A$ is the connection form for $L$ with respect to a local trivialization, then $\lambda A$ is the connection one-form which it induces on the tensor product.

We are interested in the spectral asymptotics of

$$
H(\lambda)=D(\lambda)^{*} D(\lambda)
$$

the covariant Laplacian on $L^{\lambda}$ as $\lambda \rightarrow \infty$. Let

$$
E_{1}(\lambda)=\inf (\operatorname{spec}(H(\lambda))
$$

be the bottom of the spectrum for $H(\lambda)$. Recall that $E_{1}(\lambda)$ is the infimum of the Rayleigh quotient

$$
\frac{\langle\psi, H(\lambda) \psi\rangle}{\|\psi\|^{2}}=\frac{\|D(\lambda) \psi\|^{2}}{\|\psi\|^{2}},
$$

where the infimum is taken over nonzero sections $\psi$ of $L^{\lambda}$. Here $\|\cdot\|$ denotes the $L_{2}$ norm on the space of sections of either $L^{\lambda}$ or $L^{\lambda} \otimes T^{*} M$ and $\langle\cdot, \cdot\rangle$ the inner product. The equality above is obtained by integration by parts, the boundary terms vanishing because due to Dirichlet boundary data.

We will suppose that the zero locus,

$$
C=\{B=0\} \subset M
$$


of the magnetic field is a single curve, nondegenerate in the sense that $d B \neq 0$ along this curve. If $M$ has boundary, we suppose that it is disjoint from $C$. If $M$ is not compact, we suppose that $C$ is compact, and we will suppose that the magnetic field $B$ is bounded away from zero at infinity. The next two theorems summarise our main results.

Theorem 1. Under the assumptions described in the above paragraph, $E_{1}(\lambda)=$ $O\left(|\lambda|^{2 / 3}\right)$. Specifically, there are positive constants $k$ and $K$ such that for all sufficiently large $|\lambda|$,

$$
k<\frac{E_{1}(\lambda)}{|\lambda|^{2 / 3}} \leqq K
$$

More generally, for any fixed positive integer $j$, there is a $\mathbb{A}$ sufficiently large such that for all $\lambda$ with $|\lambda| \geqq \mathbb{A}, H(\lambda)$ has at least $j$ eigenvalues $E_{j}(\lambda)$ below its continuous spectrum and they all satisfy the estimate $k<\frac{E_{j}(\lambda)}{|\lambda|^{2 / 3}} \leqq K$. The corresponding eigensections $\psi_{j}^{\lambda}$ concentrate along $C$ in the sense that for any open set $U$ whose closure is disjoint from $C$ we have

$$
\lim _{\lambda \rightarrow \infty} \int_{U}\left|\psi_{j}^{\lambda}\right|=0
$$

Remark. If $M$ is compact then there is no continuous spectrum to worry about, of course.

Remark. The last part of the theorem says that it is almost certain that the particle is very close to the zero locus $C$ provided $\lambda$ is sufficiently large. This statement can be made stronger: Let $y(m)=\operatorname{dist}(C, m)$ for $m \in M$. Then, for any exponent $v<1 / 3$ we will show that:

$$
\lim _{\lambda \rightarrow \infty} \int_{\left\{y>\lambda^{-v}\right\}}|\psi(x, \lambda)| d^{2} x=0
$$

for any family $\psi(\cdot, \lambda)$ of sections with energy of $O\left(\lambda^{2 / 3}\right)$.

Remark. The assumptions that $C$ is compact and that the magnetic field is bounded away from zero are needed to insure that the continuous spectrum grows linearly with $|\lambda|$, and so is separated from the the discrete spectra. See the end of Sect. 6.

Remark. Our analysis suggests that the eigenvalue splitting $E_{2}(\lambda)-E_{1}(\lambda)$ is $O(1)$, apparently bounded $2 \pi b_{0}^{2 / 3} /$ length $(C)^{2}$ and oscillating between this upper bound and $o(1)$. See Eq. (11) below. We plan to make this error estimate the subject of a future paper.

Theorem 1 follows rather easily from the next theorem in which we are more precise about the asymptotics at the expense of adding another assumption. We introduce the family of model operators:

$$
H_{\mathrm{anh}}(\beta)=-\frac{d^{2}}{d y^{2}}+\left(\frac{1}{2} y^{2}-\beta\right)^{2}
$$

acting on $L_{2}(\mathbb{R}) . \beta$ is a real parameter. The coordinate $y$ will be identified with the coordinate normal to $C$. Let $E(\beta)$ denote the 1st eigenvalue of $H_{\text {anh }}(\beta)$ and set

$$
E_{*}=\inf _{\beta} E(\beta)
$$


We will see that $E_{*}>0$ and $E_{*}=E\left(\beta_{*}\right)$ for some $\beta_{*}$. We have numerically calculated: $E_{*} \simeq .5698$.

Theorem 2. In addition to the assumptions made for Theorem 1, assume that the magnitude of the gradient of the magnetic field is a constant $b_{0}$ along $C$. Then

$$
\frac{E_{1}(\lambda)}{|\lambda|^{2 / 3}}=E_{*}+o(1)
$$

The same estimate holds for all eigenvalues, as in Theorem 2.

Theorems 1 and 2 should be compared with the following theorem which is a restatement of Eq. (1).

Theorem 3. Suppose that the magnetic field satisfies $|B| \geqq B_{0}$ for some positive constant $B_{0}$. Then

$$
E_{1}(\lambda) \geqq B_{0}|\lambda|
$$

Remark. If $M$ is compact without boundary and $B_{0}$ is positive, then the ground states of Theorem 3 are precisely the holomorphic sections. The multiplicity of the ground state energy is given by the Riemann-Roch theorem for large charge. In particular, the ground state is always degenerate for sufficiently positive Chern class (total flux). Theorem 3 and these results appear to be well-known to experts. See Demailly $[7,8]$ and references therein. For completeness we prove them in our appendix.

\section{Sketch of Proofs.}

As discussed above

$$
E_{1}(\lambda)=\inf _{\phi} \frac{\|D(\lambda) \phi\|^{2}}{\|\phi\|^{2}},
$$

where the infimum is taken over all nonzero smooth sections satisfying the boundary conditions. We will refer to the function to be infimised as the Rayleigh quotient, or energy of a section. Upper bounds for $E_{1}$ can be obtained by calculating the energy of a family of test functions. Lower bounds are harder. For them we rely on a partition of unity trick for splitting up the Laplacian known as IMS localization and discussed in detail in $[27,6]$.

Remark. The theorem of Simon in [27] is motivated by Witten's proof of the Morse inequalities. At a certain point Witten argues that one can use WKB analysis to estimate the number of low-lying eigenvalues and Simon makes this argument rigorous. For another approach to rigorizing this argument, see Helffer [18].

We begin by proving Theorem 2 . Our proof is based on a calculation which shows that when written in normal coordinates near $C$ the Hamiltonian is close to a separable Hamiltonian $H_{0}(\lambda)$ acting on the space of functions on $C \times \mathbb{R} \simeq S^{1} \times \mathbb{R}$. After separating variables, the part of $H_{0}$ acting normal to $C$ becomes an anharmonic oscillator Hamiltonian. This can be analyzed precisely and leads to our estimates.

Most of the technical tools for our proof are borrowed from B. Simon's article "Semiclassical Analysis of Low lying Eigenvalues I: Nondegenerate minima," [27]. The main tool is the so-called IMS localization method which lets us localize the 
Hamiltonian near $C$. This is done by multiplying wave functions by a family $J_{1}^{\lambda}$ of nonnegative functions whose support contains $C$ and shrinks to $C$ as $\lambda \rightarrow \infty$. The upper bound of Theorem 1 is proved by inserting functions of the form $J_{1}^{\lambda} \phi$, where the $\phi$ are eigensections for $H_{0}$ into the Rayleigh quotient. The lower bound follows from the IMS localization formula (28) below. This is a formula for $H$ in terms of $J_{1} H J_{1}$, a term supported away from $C$, and some corrections.

Concentration of eigensections follows from Theorem 3 by contradiction. Roughly: If a family $\psi^{\lambda}$ of sections is supported away from $C$, then its energy must grow linearly, like $\lambda B_{0}$, where $B_{0}>0$ is a lower bound for the absolute value of the magnetic field on the support of the family of sections.

The estimate of Theorem 1 follows from Theorem 2 by perturbing the metric near $C$ in order to obtain a new metric for which the hypothesis of Theorem 2 holds. We follow how the perturbation changes the Rayleigh quotient and this leads to the necessary estimates.

Theorem 3 is proved in the Appendix.

\section{Deriving the Anharmonic Oscillator}

4.1. A Model Case: Rotationally Symmetric Field. The mechanism at work behind our theorems can be seen most simply by assuming that the magnetic field is rotationally symmetric. Let $r, \theta$ be polar coordinates on the plane and suppose that

$$
A=A_{\theta}(r) d \theta
$$

is the potential for such a magnetic field:

$$
B(r)=\frac{1}{r} \frac{d}{d r} A_{\theta}(r)
$$

Expressed in polar coordinates, the Hamiltonian (5) is

$$
H=-\frac{1}{r} \frac{\partial}{\partial r}\left(r \frac{\partial}{\partial r}\right)-\frac{1}{r^{2}}\left(\frac{\partial}{\partial \theta}-i \lambda A_{\theta}(r)\right)^{2} .
$$

We suppose that there is a unique $r=r_{0}>0$ such that

$$
B\left(r_{0}\right)=0
$$

and

$$
\frac{d B}{d r}\left(r_{0}\right) \neq 0
$$

Then the zero locus $C$ is the circle of radius $r_{0}$ and

$$
A_{\theta}=A_{1}+\frac{1}{2} b(r)\left(r-r_{0}\right)^{2}
$$

where $b\left(r_{0}\right) \neq 0$ and $2 \pi A_{1}$ is the total flux, or holonomy, enclosed by $C$. We can separate variables by writing

$$
\psi(r, \theta)=\exp (i k \theta) \phi(r)
$$


with $k$ an integer. Then

$$
H \psi=\left(H_{k}(\lambda) \phi\right) \exp (i k \theta),
$$

where

$$
H_{k}(\lambda)=-\frac{1}{r} \frac{d}{d r}\left(r \frac{d \phi}{d r}\right)+\lambda^{2} \frac{1}{r^{2}}\left\{(k / \lambda)-A\left(r_{0}\right)-\frac{1}{2} b(r)\left(r-r_{0}\right)^{2}\right\}^{2} \phi .
$$

Set

$$
V=V_{k, \lambda}=\frac{1}{r^{2}}\left\{(k / \lambda)-A\left(r_{0}\right)-\frac{1}{2} b(r)\left(r-r_{0}\right)^{2}\right\}^{2} .
$$

Our reduced Hamiltonian,

$$
H_{k}(\lambda)=-\left(\frac{d}{d r}\right)^{2}+\lambda^{2} V(r)+\frac{d}{d r} \text { term }
$$

has the form of a Hamiltonian for a one-dimensional particle in a strongly constraining potential field. Setting

$$
\beta(k, \lambda)=\left(k / \lambda r_{0}\right)-\left(A_{1} / r_{0}\right)
$$

and $\frac{1}{r_{0}}\left(r-r_{0}\right)=y$ we see that

$$
V \sim\left(\beta(k, \lambda)-b\left(r_{0}\right) \frac{1}{2} y^{2}\right)^{2},
$$

so that our Hamiltonian is roughly of the form of a strong anharmonic oscillator:

$$
H \sim \frac{d^{2} \phi}{d y^{2}}+\lambda^{2}\left(\frac{1}{2} y^{2}-\beta\right)^{2}
$$

The WKB method now suggests our theorems. The exponent $2 / 3$ in front of the energy can be derived by a scaling argument which reduces the above operator to a standard anharmonic oscillator Hamiltonian.

4.2. General Case: Normal Coordinates. Introduce normal coordinates $(x, y)$ in a neighborhood of the zero locus $C$ with $x$ arc length along $C$ and $y$ normal to $C$. Then $C=\{y=0\}$ and the metric near $C$ has the form

$$
d s^{2}=f(x, y)^{2} d x^{2}+d y^{2}
$$

where

$$
f(x, 0)=1
$$

The area form is

$$
d^{2} x=f d x \wedge d y .
$$

We can choose a local gauge (section) so that

$$
A=A_{x}(x, y) d x=\left(A_{1}+\frac{1}{2} b(x, y) y^{2}\right) d x .
$$


If $L$ denotes the length of $C$, then $A_{1}=\frac{1}{i L} \log (H o l o n o m y(C))=\frac{1}{L} \int_{C} A$, and $b(x, 0)=$ $\nabla B(x, 0) \cdot($ normal $)=\frac{\partial B}{\partial y}(x, 0) \neq 0$, and

$$
H(\lambda)(\psi)=-\frac{1}{f}\left(\frac{\partial}{\partial y} f \frac{\partial \psi}{\partial y}\right)-\frac{1}{f}\left(\frac{\partial}{\partial x}-i \lambda A_{x}\right)\left[\frac{1}{f^{2}}\left(\frac{\partial \psi}{\partial x}-i \lambda A_{x} \psi\right)\right] .
$$

To obtain the approximate operator $H_{0}(\lambda)$ we replace $f(x, y)$ by $f(x, 0)=1$ and $b(x, y)$ by $b_{0}= \pm|\nabla B(x, 0)|$, assumed constant.

$$
H_{0}(\lambda)=-\frac{\partial^{2}}{\partial y^{2}}-\left(\frac{\partial}{\partial x}-i \lambda\left(A_{1}+\frac{1}{2} b_{0} y^{2}\right)\right)^{2} .
$$

We expect

$$
H(\lambda) \sim H_{0}(\lambda)
$$

for small $y$. A significant part of the proof concerns being precise about the meaning of " "

We can think of $H_{0}(\lambda)$ as a self-adjoint operator acting on $L^{2}\left(S^{1} \times \mathbb{R}\right)$, where the circle $S^{1}$ has length $L$ and is coordinatized by $x$. Since $H_{0}$ commutes with $\frac{\partial}{\partial x}$ we can separate variables. Set

$$
\psi=\frac{1}{\sqrt{L}} e^{\frac{12 \pi k x}{L}} \varphi(y)
$$

Then

$$
H_{0} \psi=\frac{1}{\sqrt{L}} e^{\frac{i 2 \pi k \lambda}{L}}\left\{\frac{-d^{2} \varphi}{d y^{2}}+\lambda^{2} b_{0}^{2}\left(\frac{2 \pi k}{\lambda L b_{0}}-\frac{A_{1}}{b_{0}}-\frac{1}{2} y^{2}\right)^{2} \varphi\right\} .
$$

The operator on $\varphi$ has the form of an anharmonic oscillator.

$$
H_{\mathrm{anh}}(\lambda ; \beta) \varphi=\frac{-d^{2} \varphi}{d y^{2}}+\lambda^{2}\left(\beta-\frac{1}{2} y^{2}\right)^{2} \varphi
$$

with a strong potential $(\lambda \gg 0)$. Here we have replaced $\lambda b_{0}$ by $\lambda$ and set

$$
\beta=\beta(k, l)=\frac{2 \pi k}{\lambda L b_{0}}-\frac{A_{1}}{b_{0}}
$$

\section{The Anharmonic Oscillator Family}

The dilation

$$
(T(\alpha) \phi)(y)=\sqrt{\alpha} \phi(\alpha y)
$$

is a unitary operator on $L^{2}(\mathbb{R}, d y)$ which satisfies $\frac{d}{d y} T(\alpha)=\alpha T(\alpha) \frac{d}{d y}$ and $y^{s} T(\alpha)=$ $\alpha^{-s} T(\alpha) y^{s}$. By computing $T(\alpha) H_{\text {anh }}(\lambda ; \beta)$ and balancing the strengths of the kinetic and potential terms of the resulting Hamiltonians we obtain the scaling law

$$
H_{\mathrm{anh}}(\lambda ; \beta) T\left(\lambda^{\frac{1}{3}}\right)=T\left(\lambda^{\frac{1}{3}}\right) \lambda^{\frac{2}{3}} H_{\mathrm{anh}}\left(1, \beta \lambda^{\frac{2}{3}}\right) .
$$


Let $E(\beta)$ be the lowest eigenvalue of $H_{\mathrm{anh}}(1, \beta)$. Then

$$
E_{1}(\lambda ; \beta)=\lambda^{\frac{2}{3}} E\left(\beta \lambda^{\frac{2}{3}}\right),
$$

where $E_{1}(\lambda, \beta)$ is the lowest eigenvalue of $H_{\text {anh }}(\lambda ; \beta)$.

For $\beta=-|\beta|$ negative we have

$$
H_{\mathrm{anh}}(1, \beta)=-\frac{d^{2}}{d y^{2}}+\left(\frac{1}{2} y^{2}+|\beta|\right)^{2}>\beta^{2} .
$$

For $\beta$ positive the potential is a "seagull potential." It can be factored: $\left(\frac{1}{2} y^{2}-\beta\right)^{2}=$ $\left(\frac{1}{\sqrt{2}} y-\sqrt{\beta}\right)^{2}\left(\frac{1}{\sqrt{2}} y+\sqrt{\beta}\right)^{2}$. Consequently, for $\beta \gg 1, H_{1}(1, \beta)$ is approximately two widely separated harmonic oscillators each of frequency $2 \sqrt{\beta}$. Thus for positive $\beta$ we have

$$
H_{\mathrm{anh}}(1 ; \beta)>4 \sqrt{\beta}+o(\sqrt{\beta}) .
$$

(See Simon [27] for a rigorous proof of this bound.) In either case $E(\beta)$ is positive and unbounded for large $|\beta|$. Since $E(\beta)$ is a continuous function of $\beta$, its infimum

$$
E_{*}=\inf _{\beta} E(\beta)
$$

is a positive number which is realized for some $\beta_{*}, \beta_{*},<0$,

$$
E_{*}=E(\beta)
$$

Numerically, we have found

$$
E_{*} \simeq .5698
$$

and $\beta_{*} \simeq .730$. (Thank you, Cesar Castilho)

Returning to our model operator $H_{0}(\lambda)$ on $L^{2}\left(S^{1} \times \mathbb{R}\right)$ we see that

$$
H_{0}(\lambda) \geqq \lambda^{\frac{2}{3}} b_{0}^{\frac{2}{3}} E_{*} .
$$

Moreover, if $k=k(\lambda)$ is any sequence of integers such that

$$
\lambda^{\frac{2}{3}}\left(\frac{2 \pi k(\lambda)}{\lambda L b_{0}}-\frac{A_{1}}{b_{0}}\right) \rightarrow \beta_{*}
$$

then

$$
\lim \frac{1}{\lambda^{\frac{2}{3}}} E_{1}\left(H_{0}(\lambda ; k(\lambda))\right)=E_{*} .
$$

Here we use $E_{1}(H)$ to denote the first eigenvalue of an operator $H$, and $H_{0}(\lambda, k)$ is the restriction of $H_{0}(\lambda)$ to the space with angular momentum $k$. Our sequence $k(\lambda)$ satisfies

$$
\frac{2 \pi k(\lambda)}{\lambda b_{0} L}=\frac{1}{b_{0}} A_{1}+\beta_{*} \lambda^{\frac{-2}{3}}+o\left(\lambda^{\frac{-2}{3}}\right) .
$$


(It is a straightforward exercise in number theory to see that there is a sequence $k(\lambda)$ of integers, $\lambda$ integer, satisfying this property.) The corresponding family of ground states for $H_{0}(\lambda, k(\lambda))$ is

$$
\phi(y ; \lambda)=\lambda^{\frac{1}{6}} \phi_{1}\left(\lambda^{\frac{1}{3}} y\right),
$$

where $\phi_{1}$ is the positive normalized ground state for

$$
\frac{-d^{2}}{d y^{2}}+\left(\beta(k, \lambda)-\frac{1}{2} y^{2}\right)^{2}=\frac{-d^{2}}{d y^{2}}+\left(\beta_{*}+o(1)-\frac{1}{2} y^{2}\right)^{2} \text {. }
$$

One can work out its asymptotics using the WKB method. We will not need the full asymptotics but only the bounds:

$$
\begin{aligned}
\left|\phi_{1}(y)\right| \leqq \frac{c}{|y|} e^{-\frac{1}{6}(1-\delta)|y|^{3}}, \\
\left|\frac{d \phi_{1}}{d y}(y)\right| \leqq c y^{2} e^{-\frac{1}{6}(1-\delta)|y|^{3}},
\end{aligned}
$$

valid for all $y$ with $|y|$ greater than some $Y_{0}(c, \delta)$. Here $\delta$ is an arbitrary small positive number, and $c$ is a positive constant. For rigorous proofs of these WKBtype bounds see [21] or [9].

Remark on higher eigenfunctions for $H_{0}$. Observe that if $k(\lambda)$ satisfies the condition of Eq. 8, then so does any sequence of the form $k(\lambda)+\delta k$ for any fixed integer $\delta k$. The corresponding functions $\psi$ of the ansatz are orthogonal since $\exp (i 2 \pi k x / L)$ and $\exp (i 2 \pi((k+\delta k) x / L)$ are orthogonal for $\delta k$ a nonzero integer.

Using perturbation theory we can estimate the size of the eigenvalue splitting:

$$
\delta E=E_{1}(k(\lambda)+\delta k, \lambda)-E_{1}(k(\lambda, k)
$$

due to such a change of wave number. Since $\beta_{*}$ minimizes $E(\beta)$ we have $d E /\left.d \beta\right|_{\beta_{*}}=0$. And

$$
\delta \beta=\beta(k+\delta k, \lambda)-\beta(k, \lambda)=2 \pi \delta k / \lambda L .
$$

It follows that

$$
\begin{aligned}
\delta E & \left.\sim \lambda^{2 / 3} b_{0}^{2 / 3} \frac{1}{2} \frac{d^{2} E}{d \beta^{2}}\right|_{\beta_{*}}\left(\lambda^{2 / 3} \delta \beta\right)^{2} \\
& =(\delta k)^{2} b_{0}^{2 / 3}\left(\frac{2 \pi}{L}\right)^{2} \\
& =O(1) .
\end{aligned}
$$

This proves that for each fixed $j$ the $j^{\text {th }}$ eigenvalue $E_{j}^{0}(\lambda)$ of $H_{0}$ also satisfies

$$
\lim \frac{E_{J}^{0}(\lambda)}{\lambda^{2 / 3}}=b_{0}^{2 / 3} E_{*}
$$


In particular, it also implies the eigenvalue splitting estimate

$$
E_{2}^{0}(\lambda)-E_{1}^{0}(\lambda) \leqq(1+o(1)) b_{0}^{2 / 3}\left(\frac{2 \pi}{L}\right)^{2}=O(1)
$$

The WKB bounds $(9,10)$ for the ground state also hold also for these $j^{\text {th }}$ eigenfunctions.

\section{Proof of Theorem 1: Upper Bound}

6.1. Upper Bound. We establish the upper bound

$$
\lim \sup \frac{E_{1}(\lambda)}{\lambda^{2 / 3}} \leqq b_{0}^{2 / 3} E_{*}
$$

by inserting test functions of the form

$$
\psi^{\lambda}=J_{1}^{\lambda}(y) \psi(x, y ; \lambda)
$$

into the variational principle (6) for $E_{1}(\lambda)$. Here

$$
\psi(x, y ; \lambda)=\frac{1}{\sqrt{L}} e^{\frac{2 \pi k k(\lambda) x}{L}} \phi_{0}(y ; \lambda) .
$$

are the ground states for $H_{0}(\lambda)$ as described in the previous two sections. Thus the $\phi_{0}(y ; \lambda)$ are ground states for our anharmonic oscillator family. The functions $J_{1}^{\lambda}$ are a family of bump functions centered on the curve $C=\{y=0\}$ whose width shrinks to zero as $\lambda \rightarrow \infty$ in a manner which we now describe.

Introduce a fixed smooth function $J_{1}(y)$ of $y$ whose values lie between 0 and 1 and which is identically 1 for $|y| \leqq 1$, and identically 0 for $|y| \geqq 2$. Define the function $J_{1}^{\lambda}$ by

$$
J_{1}^{\lambda}(y):=J_{1}\left(\lambda^{v} y\right) \text {. }
$$

The exponent $v$ must satisfy

$$
2 / 9<v<1 / 3 .
$$

This inequality is crucial due to the fact that

$$
\lambda^{2 / 9} \ll \lambda^{v} \ll \lambda^{1 / 3}
$$

as $\lambda \rightarrow \infty$ (cf. with the WKB scaling at the end of Sect. 4).

The upper bound (12) follows from the following three lemmas.

Lemma 1. The ansätz $\psi^{\hat{\lambda}}$ satisfies

$$
\left\|\psi^{\lambda}\right\|=1+o(1)
$$

as $\lambda \rightarrow \infty$.

Set

$$
D_{0}(\lambda) \psi=\left(\frac{\partial}{\partial y} \psi\right) d y=\left(\frac{\partial}{\partial x} \psi-i \lambda\left(A_{1}+b_{0} \frac{1}{2} y^{2}\right) \psi\right) d x
$$


for $\psi$ a section defined near $C$. If the adjoint ${ }^{*}$ is taken with respect to the flat metric on $C \times \mathbb{R}=S^{1}(L) \times \mathbb{R}$, then our model operator is given as

$$
H_{0}=D_{0}^{*} D_{0}
$$

\section{Lemma 2.}

$$
\left\|D_{0} \psi^{\lambda}\right\|^{2} \leqq \lambda^{2 / 3} b_{0}^{2 / 3} E_{*}+o\left(\lambda^{2 / 3}\right)
$$

Lemma 3. There exists a positive constant $c$ such that for all sufficiently large $\lambda$,

$$
\left|\left\|D\left(J_{1}^{\lambda} \psi^{\lambda}\right)\right\|^{2}-\left\|D_{0}\left(J_{1}^{\lambda} \psi^{\lambda}\right)\right\|^{2}\right| \leqq c\left\{\lambda^{1-3 v} \| D^{0}\left(J_{1}^{\lambda} \psi^{\lambda}\|\| J_{1}^{\lambda} \psi\left\|+\lambda^{2-6 v}\right\| J_{1}^{\lambda} \psi \|\right\} .\right.
$$

Let us see how the upper bound follows from these lemmas. Combining them and recalling that $\psi^{\lambda}=J_{1}^{\lambda} \psi_{0}(x, y ; \lambda)$ we see that

$$
\begin{aligned}
\left|\left\|D(\lambda) \psi^{\lambda}\right\|^{2}-\left\|D_{0}(\lambda) \psi^{\lambda}\right\|^{2}\right| \leqq & c \lambda^{1-3 v}\left[\lambda^{1 / 3} b_{0}^{1 / 3} \sqrt{E_{*}}+o\left(\lambda^{1 / 3}\right)\right][1+o(1)] \\
& +c \lambda^{2-6 v}[1+o(1)] \\
\leqq & o\left(\lambda^{\frac{4}{3}-3 v}+\lambda^{2-6 v}\right) .
\end{aligned}
$$

But $\frac{4}{3}-3 v, 2-6 v<2 / 3$ since $v>2 / 9$. Therefore

$$
\left|\left\|D \psi^{\lambda}\right\|^{2}-\left\|D_{0} \psi^{\lambda}\right\|^{2}\right|=o\left(\lambda^{2 / 3}\right)
$$

Then

$$
\begin{aligned}
\frac{\left\|D(\lambda) \psi^{\lambda}\right\|^{2}}{\left\|\psi^{\lambda}\right\|^{2}} & \leqq\left\|D(\lambda) \psi^{\lambda}\right\|^{2}(1+o(1)) \\
& \leqq\left[\left|\left\|D(\lambda) \psi^{\lambda}\right\|^{2}-\left\|D_{0}(\lambda) \psi^{\lambda}\right\|^{2}\right|+\left\|D_{0}(\lambda) \psi^{\lambda}\right\|^{2}\right][1+o(1)] \\
& =o\left(\lambda^{2 / 3}\right)+\lambda^{2 / 3} b_{0}^{2 / 3} E_{*}+o\left(\lambda^{2 / 3}\right),
\end{aligned}
$$

which establishes the upper bound.

Proof of Lemma 1. The WKB eigenfunction $\phi_{0}(y ; \lambda)$ is normalized:

$$
1=\int_{-\infty}^{\infty}\left|\phi_{0}(y ; \lambda)\right|^{2} d y=\int_{-\infty}^{\infty}\left(J_{1}^{\lambda}(y)\right)^{2}\left|\phi_{0}(y ; \lambda)\right|^{2} d y+\int_{-\infty}^{\infty}\left(1-\left(J_{1}^{\lambda}(y)\right)^{2}\right)\left|\phi_{0}(y ; \lambda)\right|^{2} d y .
$$

On the other hand,

$$
\left\|\psi^{\lambda}\right\|^{2}=\int\left(J_{1}^{\lambda}(y)\right)^{2} \frac{1}{L}\left|\phi_{0}(y ; \lambda)\right|^{2} f(x, y) d x d y .
$$

The support of $J_{1}^{\lambda}$ is $|y| \leqq 2 \lambda^{-v}$ and on this domain

$$
|1-f| \leqq c|y|
$$

for some constant $c$ (related to the curvature of $C$ and of the metric near $C$ ). It follows that

$$
\left.\left|\int_{-\infty}^{\infty}\left(J_{1}^{\lambda}\right)^{2}\right| \phi_{0}(y ; \lambda)\right|^{2}\left|d y-\left\|\psi^{\lambda}\right\|^{2}\right| \leqq c \int_{-2 / \lambda^{\prime}}^{2 / \lambda^{\prime}}\left(J_{1}^{\lambda}(y)\right)^{2}\left|\phi_{0}(y ; \lambda)\right|^{2}|y| d y
$$


and so

$$
\left|1-\left\|\psi^{\lambda}\right\|^{2}\right| \leqq c \int_{-2 / \lambda^{\prime}}^{2 / \lambda^{\prime \prime}}\left(J_{1}^{\lambda}(y)\right)^{2}\left|\phi_{0}(y ; \lambda)\right|^{2}|y| d y+\int\left(1-\left(J_{1}^{\lambda}\right)^{2}\right)\left|\phi_{0}(y ; \lambda)\right|^{2} d y .
$$

Now change variables to $\xi=\lambda^{1 / 3} y$ to obtain:

$$
\int_{2 / \lambda^{r^{\prime}}}^{-2 / \lambda^{\prime}}\left(J_{1}^{\lambda}(y)\right)^{2}\left|\phi_{0}(y ; \lambda)\right|^{2}|y| d y \leqq \int_{-2 \lambda^{1 / 3-r}}^{2 \lambda^{1 / 3-r}}\left|\phi_{0}(y ; \lambda)\right|^{2} \lambda^{-1 / 3}|\xi| d \xi=O\left(\lambda^{-1 / 3}\right) .
$$

The support of $1-J_{1}^{\lambda}(y)^{2}$ is $|y|>\lambda^{-v}$ so that

$$
\int_{-\infty}^{\infty}\left(1-J_{1}^{\lambda}(y)^{2}\right)\left|\phi_{0}(y ; \lambda)\right|^{2} d y \leqq 2 \int_{1 / \lambda^{v}}^{\infty}\left|\phi_{0}(y ; \lambda)\right|^{2} d y .
$$

Using the WKB estimate (9) for $\phi_{1}$ and Watson's lemma (cf. Erdelyi, or Copson [5]) we find that for any $\delta>0$ we have

$$
\int_{1 / \lambda^{v}}^{\infty}\left|\phi_{0}^{\lambda}(y)\right|^{2} d y=\int_{\lambda^{1 / 3-v}}^{\infty}\left|\phi_{1}(\xi)\right|^{2} d \xi \leqq 2 \frac{\exp \left(-\frac{1}{3}(1-\delta) \lambda^{1 / 3-v}\right)}{\lambda^{2 / 3-v}},
$$

which is much smaller than $O\left(\lambda^{-1 / 3}\right)$. Putting these inequalities together we obtain the normalization equation (13).

Proof of Lemma 3. If a section $\psi$ is supported in a small neighborhood then we can compute $D_{0}(\lambda) \psi$ and $D(\lambda) \psi$ and compare them. Now the connection one-form for $D$ is

$$
A=\left[A_{1}+\left(\frac{1}{2}\right) b_{0} y^{2}+a_{3}(x, y) y^{3}\right] d x,
$$

where $a_{3}$ is a smooth bounded function in a neighborhood of $C$. It follows that

$$
D(\lambda)=D_{0}(\lambda)+i \lambda a_{3}(x, y) y^{3} d x,
$$

so that we have the pointwise equality:

$$
\begin{aligned}
|D \psi|^{2}-\left|D_{0} \psi\right|^{2} & =\left|D_{x} \psi\right|^{2}-\left|D_{0 x} \psi\right|^{2} \\
& \left.=-\lambda f^{-2}\left\{\left(D_{0 x} \psi, i a_{3} y^{3} \psi\right)+\left(i a_{3} y^{3} \psi, D_{0 x} \psi\right)\right\}+\lambda^{2}\left(a_{3} y^{3}\right)^{2}|\psi|^{2}\right\} .
\end{aligned}
$$

(The subscripted $x \mathrm{~s}$ denote the $d x$ components of the differentials. The length of $d x$ is $f^{-1}$.) Integrating, and applying Cauchy-Schwartz we obtain

$$
\left|\|D \psi\|^{2}-\left\|D_{0} \psi\right\|^{2}\right| \leqq 2 \lambda\left\|D_{0 x} \psi\right\|\left\|\mid a_{3} y^{3} \psi\right\|+\lambda^{2}\left\|a_{3}^{2} y^{6} \psi\right\|^{2} .
$$

Now replace $\psi$ by $J_{1}^{\lambda} \psi$. (We think of multiplication by $J_{1}^{\lambda}$ as a map from the space of square integrable sections over to the space of such sections over $C \times \mathbb{R}$.) The support of $J_{1}^{\lambda} \psi$ and $D_{x}^{0} \psi^{\lambda}$ is $\left\{|y| \leqq 2 \lambda^{-v}\right\}$ so that the above inequality becomes

$\left|\left(\left\|D\left(J_{1}^{\lambda} \psi\right)\right\|\right)^{2}-\left(\left\|D_{0}\left(J_{1}^{\lambda} \psi\right)\right\|\right)^{2}\right| \leqq c_{1}\left\{\lambda^{1-3 v}\left\|D_{0}\left(J_{1}^{\lambda} \psi\right)\right\|\left\|J_{1}^{\lambda} \psi\right\|+\lambda^{2-6 v}\left(\left\|J_{1}^{\lambda} \psi\right\|\right)^{2}\right\}$, 
where the constant $c_{1}$ is independent of $\lambda$ and involves bounds on $f$ and $a_{3}$ in an arbitrarily small neighborhood of $C$, and various powers of 2. QED.

Proof of Lemma 2. It suffices to prove the stated asymptotics for the integral of

$$
\left|D_{0}(\lambda) \psi^{\lambda}\right|^{2} d x d y \text {. }
$$

This is because $|1-f| \leqq c y$ in an annular neighborhood of $C$, and the support of $D_{0} \psi^{\lambda}$ is $\left\{|y| \leqq 2 / \lambda^{\nu}\right\}$, so by an argument given previously

$$
\left.\left|\int\right| D_{0} \psi^{\lambda}\right|^{2} d x d y-\int\left|D_{0} \psi^{\lambda}\right|^{2} f d x d y \mid \leqq c^{\prime} \lambda^{-v} \int\left\|D_{0} \psi^{\lambda}\right\| d x d y
$$

for some constant $c^{\prime}$. But if

$$
\int\left\|D_{0} \psi^{\lambda}\right\| d x d y
$$

has the stated $O\left(\lambda^{2 / 3}\right)$ asymptotics, then the above inequality yields a difference which is $0\left(\lambda^{2 / 3-v}\right)=o\left(\lambda^{2 / 3}\right)$ so that the limits of $\frac{1}{\lambda^{2 / 3}} \int\left\|D_{0} \psi^{\lambda}\right\|^{2} f d x d y$ and $\frac{1}{\lambda^{2 / 3}} \int D_{0} \psi^{\lambda} \|^{2} d x d y$ are equal.

Now

$$
D_{0}\left(J_{1}^{\lambda} \psi\right)=d J_{1}^{\lambda} \otimes \psi+J_{1}^{\lambda} D_{0} \psi
$$

so that

$$
\left|D_{0}\left(J_{1}^{\lambda} \psi\right)\right|^{2}=\left|d J^{\lambda}\right|^{2}|\psi|^{2}+2 \operatorname{Re}\left(J_{1}^{\lambda} d J_{1}^{\lambda} \psi, D_{0} \psi\right)+\left(J_{1}^{\lambda}\right)^{2}\left|D_{0} \psi\right|^{2}
$$

We analyze the integral of each of these three terms separately.

First Term. The support of $d J_{1}^{\lambda}$ is $\left\{\frac{1}{\lambda^{v}} \leqq|y| \leqq \frac{2}{\lambda^{v}}\right\}$ and $d J_{1}^{\lambda}=\lambda^{v} J_{1}^{\prime}\left(\lambda^{v} y\right) d y$ (recall: $\left.J_{1}^{\lambda}(y)=J_{1}\left(\lambda^{v} y\right)\right)$ so that

$$
\int\left|d J_{1}^{\lambda} \| \psi\right|^{2} d x d y \leqq \lambda^{v} c^{\prime} \int_{\frac{1}{\lambda^{N}}}^{\frac{2}{\lambda^{2}}}|\psi|^{2} d x d y .
$$

Since $\psi$ is square integrable with integral close to 1 we obtain

$$
\int\left|d J_{1}^{\lambda} \| \psi\right|^{2} d x d y=O\left(\lambda^{2 v}\right)=o\left(\lambda^{2 / 3}\right) .
$$

(Further analysis using the WKB estimates shows that this integral is actually exponentially small in $\lambda$.)

Second Term. Assuming the validity of the $O\left(\lambda^{2 / 3}\right)$ bound on the third term we have

$$
\begin{aligned}
\left|\int\left(J_{1}^{\lambda} d J_{1}^{\lambda} \psi, D_{0} \psi\right) d x d y\right| & \leqq \sqrt{\int\left|J_{1}^{\lambda} d J_{1}^{\lambda} \psi\right|^{2} d x d y} \sqrt{\int\left|D_{0} \psi\right| d x d y} \\
& \leqq c^{\prime} \lambda^{v} \lambda^{1 / 3} b_{0}^{1 / 3} E_{*}^{1 / 2} \\
& =o\left(\lambda^{2 / 3}\right) .
\end{aligned}
$$

Third Term. $\psi=\psi(x, y ; \lambda)$ is an exact eigenvector for $H_{0}(\lambda)$ so that

$$
\int\left|D_{0} \psi\right|^{2} d x d y=\left(\lambda b_{0}\right)^{2 / 3} E_{1}(\beta(\lambda, k(\lambda)),
$$


where $E_{1}\left(\beta(\lambda, k(\lambda)) \rightarrow E_{*}\right.$ as $\lambda \rightarrow \infty$. But:

$$
\int\left|D_{0} \psi\right|^{2} d x d y=\int\left(J_{1}^{\hat{\lambda}}\right)^{2}\left|D_{0} \psi\right|^{2} d x d y+\int\left(1-\left(J_{1}^{\lambda}\right)^{2}\right)\left|D_{0} \psi\right|^{2} d x d y .
$$

The support of $1-\left(J_{1}^{\lambda}\right)^{2}$ is $\left\{|y|>\frac{1}{\lambda^{N}}\right\}$ so that

$$
\int\left(1-\left(J_{1}^{\lambda}\right)^{2}\right)\left|D_{0} \psi\right|^{2} d x d y \leqq 2 \int_{1 / \lambda^{V}}^{\infty}\left|D_{0} \psi\right|^{2} d x d y
$$

The latter integral is analyzed using the WKB estimate (10) for $\phi_{1}$ and Watson's lemma ([5 or 10]). One finds it to be of the form $O\left(P(\lambda) \exp \left(-\frac{1}{3} \lambda^{1-3 v}\right)\right)$ for a polynomial $P$. In particular:

$$
\int\left(1-\left(J_{1}^{\lambda}\right)^{2}\right)\left|D_{0} \psi\right|^{2} d x d y=o\left(\lambda^{2 / 3}\right)
$$

Integrating Eq. (20) and using Eq. (21)-(25) yields the claimed result.

6.2. Higher Eigenvalues. A slight extension of the above argument, applied to the $j^{\text {th }}$ eigenfunctions of $H_{0}$ in place of its ground state, gives the same upper bound for the $j^{\text {th }}$ eigenvalue $E_{j}(\lambda)$, for all $j$.

A priori, it is not apparent that there is any discrete spectrum. We remedy this by combining Theorem 3 with Perrson's theorem ([6], p. 38) which states that the infimum of the essential spectrum of $H(\lambda)$ is given by the min-max expression:

$$
\sup _{K} \inf f_{\{\psi: \operatorname{supp}(\psi) \subset M \backslash K\}} \frac{\langle\psi, H(\lambda) \psi\rangle}{\|\psi\|^{2}} .
$$

Here $K$ ranges over the compact subsets of $M$. According to Theorem 3, this last quantity is bounded below by $|\lambda| B_{0}$, where $B_{0}$ is a lower bound for the absolute value of the magnetic field as we approach infinity (cf. Remark 2 following the aforementioned statement of Perrson's theorem, and Theorem 6.1 of that reference). In particular, the essential spectrum must grow at least linearly, so there is plenty of room for eigenvalues growing like $\lambda^{2 / 3}$.

The extension to the higher eigenvalues now follows the lines of Simon's article, using the observation made at the end of our Sect. 5. There we observed that for each integer $\delta k$, the $H_{0}$-eigenfunctions corresponding to two different sequences $k(\lambda)+\delta k, k(\lambda)+\delta j, \delta k \neq \delta j, \lambda, \delta j, \delta k=1,2,3, \ldots$ are orthonormal relative to the norm for sections over $C \times \mathbb{R}$ (product metric) and that the corresponding $H_{0}(\lambda)$-eigenvalues $E_{J}^{0}(\lambda)$ satisfy $\lim \frac{E_{J}^{0}(\lambda)}{\lambda^{2 / 3}}=b_{0}^{2 / 3} E_{*}$. The sections obtained by multiplying these $H_{0}$ eigensections by $J_{1}^{\lambda}$ are easily shown to satisfy Lemmas 1 and 2 , and hence the first $j$ eigenvalues are less than $\lambda^{2 / 3} b_{0}^{2 / 3} E_{*}+o\left(\lambda^{2 / 3}\right)$.

\section{Proof of Theorem 1: Lower Bound}

We will show that

$$
H(\lambda) \geqq \lambda^{2 / 3} b_{0}^{2 / 3} E
$$


for any $E<E_{*}$. It follows immediately that $\lim \inf \frac{1}{\lambda^{2 / 3}} E_{1}(\lambda) \geqq b_{0}^{2 / 3} E_{*}$, the desired result. Set

$$
J_{0}^{\lambda}=\sqrt{1-\left(J_{1}^{\lambda}\right)^{2}}
$$

with $J_{1}^{\hat{\lambda}}$ as in the previous section. The proof of Eq. (26) is based on the IMS localization formula for $H$ :

$$
H(\lambda)=J_{1}^{\lambda} H(\lambda) J_{1}^{\lambda}+J_{0}^{\lambda} H(\lambda) J_{0}^{\lambda}-\left|d J_{1}^{\lambda}\right|^{2}-\left|d J_{0}^{\lambda}\right|^{2} .
$$

See [27] or [6] regarding IMS localization.

Derivation of (28). An IMS type formula is valid for any operator of the form covariant Laplacian plus potential. (See for example the definition in [2] of generalized Laplacians.) Let $f$ denote the operator of multiplication of sections by the smooth function $f$. Then

$$
[f,[f, H]]=-2|d f|^{2},
$$

from which it follows that

$$
f H f=\frac{1}{2}\left(f^{2} H+H f^{2}\right)+|d f|^{2} .
$$

Add the $f H f$ formulas together for $f=J_{0}^{\lambda}$ and $f=J_{1}^{\lambda}$ and remember that $\left(J_{0}^{\lambda}\right)^{2}+$ $\left(J_{1}^{\lambda}\right)^{2}=1$ to obtain the IMS formula (28).

We now proceed to obtain bounds for each of the terms in the IMS formula. In the previous section we showed that

$$
\int\left|d J_{a}^{\lambda}\right|^{2}|\psi|^{2} d^{2} x \leqq \lambda^{2 v} \int|\psi|^{2} d^{2} x
$$

It follows that

$$
\left(\left|d J_{1}^{\lambda}\right|^{2}+\left|d J_{0}^{\lambda}\right|^{2}\right) \leqq 2 \lambda^{2 v}=o\left(\lambda^{2 / 3}\right),
$$

where the left-hand side is thought of as a multiplication operator. (As shown in the previous section, these terms are actually exponentially small in $\lambda$.) This bounds the last two terms of the IMS formula.

To bound the $J_{0} H J_{0}$ term consider the covariant Laplacian with Dirichlet boundary data for sections over the set $\left\{|y| \geqq \frac{1}{\lambda^{3}}\right\}$. Observe that for any smooth section $\psi$ we have $J_{0}^{\lambda} \psi=0$ on the set $\left\{|y|=\frac{1}{\lambda^{\prime}}\right\}$. So $J_{0}^{\lambda} \psi$ can be considered as in the domain of this restricted covariant Laplace operator. Now

$$
|B| \geqq b_{0} \lambda^{-v}+o\left(\lambda^{-v}\right) \text { for }|y| \geqq \frac{1}{\lambda^{v}} .
$$

It follows from Theorem 3 (i.e. the bound of Eq. (1)) that

$$
\left\langle\psi, J_{0}^{\hat{\lambda}} H(\lambda) J_{0}^{\lambda} \psi\right\rangle=\left\langle J_{0}^{\lambda} \psi, H(\lambda) J_{0}^{\lambda} \psi\right\rangle \geqq \lambda\left[\left(b_{0} \lambda^{-v}\right)+o\left(\lambda^{-v}\right)\right]\left\|\left(J_{0}^{\lambda}\right)^{2} \psi\right\|^{2}
$$

or:

$$
J_{0}^{\lambda} H(\lambda) J_{0}^{\lambda} \geqq\left[b_{0} \lambda^{1-v}+o\left(\lambda^{1-v}\right)\right]\left(J_{0}^{\lambda}\right)^{2} \gg \lambda^{2 / 3}\left(b_{0}^{2 / 3} E\right)\left(J_{0}^{\lambda}\right)^{2} .
$$

Our upper bound will be complete once we have shown that

$$
J_{1}^{\lambda} H(\lambda) J_{1}^{\lambda}>\left\{\lambda^{2 / 3} b_{0}^{2 / 3} E+o\left(\lambda^{2 / 3}\right)\right\}\left(J_{1}^{\lambda}\right)^{2} .
$$


For then, by the IMS formula (28) and (34),

$$
H(\lambda)>\lambda^{2 / 3} b_{0}^{2 / 3} E\left(J_{1}^{\lambda}\right)^{2}+\lambda^{2 / 3} b_{0}^{2 / 3} E\left(J_{2}^{\lambda}\right)^{2}+o\left(\lambda^{2 / 3}\right)=\lambda^{2 / 3} b_{0}^{2 / 3} E+o\left(\lambda^{2 / 3}\right),
$$

as required.

Equation (35) is in turn implied by

$$
J_{1}^{\lambda} H_{0}(\lambda) J_{1}^{\lambda}>\left(\lambda^{2 / 3} b_{0}^{2 / 3} E+o\left(\lambda^{2 / 3}\right)\right)\left(J_{1}^{\lambda}\right)^{2},
$$

and Lemma 3 of the previous section. For observe that the left-hand side of the inequality in that lemma is

$$
\left|\left\langle\psi, J_{1}^{\lambda}\left(H(\lambda)-H_{0}(\lambda)\right) J_{1}^{\lambda} \psi\right\rangle\right| .
$$

It follows that

$$
\begin{aligned}
\frac{1}{\lambda^{2 / 3}}\left\langle\psi, J_{1}^{\lambda} H(\lambda) J_{1}^{\lambda} \psi\right\rangle \geqq & \frac{1}{j^{2 / 3}}\left\langle\psi, J_{1}^{\lambda} H_{0}(\lambda) J_{1}^{\lambda} \psi\right\rangle \\
& -\frac{1}{, 2 / 3} c\left\{\lambda^{1-3 v}\left\|D_{0}\left(J_{1}^{\lambda} \psi\right)\right\|\left\|J_{1}^{\lambda} \psi\right\|+\lambda^{2-6 v}\left\|J_{1}^{\lambda} \psi\right\|^{2}\right\} .
\end{aligned}
$$

But

$$
\left\langle\psi, J_{1}^{\lambda} H_{0}(\lambda) J_{1}^{\lambda}\right\rangle=\left\|D_{0}(\lambda) J_{1}^{\lambda} \psi\right\|^{2},
$$

so that this inequality becomes

$$
\begin{aligned}
\frac{1}{\lambda^{2 / 3}}\left\langle\psi, J_{1}^{\lambda} H(\lambda) J_{1}^{\lambda} \psi\right\rangle \geqq & \frac{\left\|D_{0}(\lambda) J_{1}^{\lambda} \psi\right\|}{\lambda^{1 / 3}}\left\{\frac{\left\|D_{0}(\lambda) J_{1}^{\lambda} \psi\right\|}{\lambda^{1 / 3}}-c \frac{\lambda^{1-3 v}}{\lambda^{1 / 3}}\left\|J_{1}^{\lambda} \psi\right\|\right\} \\
& +\frac{\lambda^{2-6 v}}{\lambda^{1 / 3}}\left\|J_{1}^{\lambda} \psi\right\|^{2} .
\end{aligned}
$$

Now $v>\frac{2}{9}$ so that $1-3 v<\frac{1}{3}, 2-6 v<\frac{2}{3}$ and $\frac{\lambda^{1-3 v}}{\lambda^{1 / 3}}, \frac{\lambda^{2-6 v}}{\lambda^{2 / 3}}$ both go to zero as $\lambda \rightarrow \infty$. We have

$$
\frac{\left\|D_{0} J_{1}^{\lambda} \psi\right\|}{\lambda^{1 / 3}}-c \frac{\lambda^{1-3 v}}{\lambda^{1 / 3}}\left\|J_{1}^{\lambda} \psi\right\| \geqq\left\|J_{1}^{\lambda} \psi\right\|\left(\sqrt{E} b_{0}+o(1)\right),
$$

so that

$$
\frac{1}{\lambda^{2 / 3}}\left\langle\psi, J_{1}^{\lambda} H J_{1}^{\hat{\lambda}} \psi\right\rangle \geqq\left\|\left(J_{1}^{\lambda}\right) \psi\right\|^{2}\left(E b_{0}^{2 / 3}+o(1)\right)
$$

As claimed.

It remains to prove Eq. (37). Refer back to the proof of Lemma 2, (18), of the previous section where we proved

$$
\left|\left\|D_{0}\left(J_{1}^{\lambda} \psi\right)\right\|_{M}^{2}-\left\|D_{0}\left(J_{1}^{\lambda} \psi\right)\right\|_{C \times \mathbb{R}}^{2}\right|<c \lambda^{-v}\left\|D_{0}\left(J_{1}^{\hat{\lambda}} \psi\right)\right\|_{C \times \mathbb{R}}^{2},
$$

the notation of subscripted norms being obvious, we hope. We analyzed the spectrum of $H_{0}(\lambda)$ several sections ago and found that for all $\varphi,\left\|D_{0} \varphi\right\|_{C \times \mathbb{R}}^{2}>\lambda^{2 / 3} b_{0}^{2 / 3}$ $E\|\varphi\|_{C \times \mathbb{R}}^{2}$. In particular, for $\varphi=J_{1}^{\lambda} \psi$ we find that $\left\|D_{0}\left(J_{1}^{\lambda} \psi\right)\right\|_{C \times \mathbb{R}}^{2}>\lambda^{2 / 3} b_{0}^{2 / 3}$ $E\left\|J_{1}^{\lambda} \psi\right\|_{C \times \mathbb{R}}^{2}$. Finally

$\left\|J_{1}^{\lambda} \psi\right\|_{C \times \mathbb{R}}^{2} \geqq\left\|J_{1}^{\lambda} \psi\right\|_{M}^{2}-\left|\left\|J_{1}^{\lambda} \psi\right\|_{M}^{2}-\left\|J_{1}^{\lambda} \psi\right\|_{C \times \mathbb{R}}^{2}\right|>\left\|J_{1}^{\lambda} \psi\right\|_{M}^{2}+c \lambda^{-v}\left\|J_{1}^{\lambda} \psi\right\|_{M}^{2}$. 
Combining these last few inequalities yields Eq. (37).

This proves the lower bound for the ground state energy, and hence completes the proof of the energy bounds of Theorem 2 .

\section{Eigenvalue Concentration}

We will prove the following

Lemma 4. Let $\psi(\cdot, \lambda)$ be a sequence of sections of $L^{\lambda}$ such that $\frac{1}{\lambda^{2 / 3}}\langle\psi(\cdot, \lambda)$, $H(\lambda) \psi(\cdot, \lambda)\rangle$ is bounded as $\lambda \rightarrow \infty$. Then the $\psi(\cdot, \lambda)$ concentrate on $C$ in the sense that

$$
\left\|J_{0}^{\lambda} \psi(\cdot, \lambda)\right\|_{M}^{2}=o\left(\lambda^{v-1 / 3}\right) \rightarrow 0,
$$

where $J_{0}^{\lambda}$ is the function used in the proof of the lower bound. (The support of $J_{0}^{\lambda}$ is the set $\left\{|y|>\frac{1}{\lambda^{\prime}}\right\}$.)

According to the upper eigenvalue bounds of Theorem 1 and 2 , the $j^{\text {th }}$ eigensections satisfy the uniform bound of this lemma. This proves the concentration result claimed in Theorem 2.

Proof of Lemma. Let $b_{*}$ be any positive number smaller than the minimum of $|\nabla B|$ over $C$. Then for $\lambda$ sufficiently large the absolute value of the magnetic field is greater than $\frac{b_{*}}{\lambda^{\prime}}$ on the support of $J_{0}^{\hat{\lambda}}$. It follows from Theorem 3 , the nonzero magnetic field eigenvalue bound, that

$$
\left\langle\psi(\cdot, \lambda), J_{0}^{\hat{\lambda}} H(\lambda) J_{0}^{\lambda} \psi(\cdot, \lambda)\right\rangle \geqq \lambda\left(\frac{b_{*}}{\lambda^{v}}\right)\left\|J_{0}^{\lambda} \psi(\cdot, \lambda)\right\|_{M}^{2} .
$$

Combine this with the IMS localization formula (28) and the bounds on the $\left|d J_{a}^{\lambda}\right|(21)$ to obtain

$$
\frac{1}{\lambda^{2 / 3}}\langle\psi(\cdot, \lambda), H(\lambda) \psi(\cdot, \lambda)\rangle \geqq \lambda^{1 / 3-v} b_{*}\left\|J_{0}^{\lambda} \psi(\cdot, \lambda)\right\|_{M}^{2}+o(1) .
$$

On the other hand, we are given that the left-hand side of this inequality is bounded above by some constant $K$. It follows that for sufficiently large $\lambda$ we have

$$
\frac{K}{\lambda^{1 / 3-v}} \geqq\left\|J_{0}^{\lambda} \psi(\cdot, \lambda)\right\|_{M}^{2}
$$

as desired.

QED.

\section{Proof of Theorem 1}

The eigenvalue estimate of Theorem 1 follows from that of Theorem 2 and the Rayleigh-Ritz principle. First observe that we can obtain the situation of Theorem 1 from that of Theorem 2 by perturbing the metric $g$ of Theorem 2 while leaving the connection $A$ fixed. Let $x, y$ be coordinates in a neighborhood of $C$ such that (with respect to a certain local trivialization) $A=\left(A_{1}+(1 / 2) y^{2}\right) d x, x$ is an angular coordinate along $C$ and $C$ is given by $y=0$. (These are no longer normal coordinates 
with respect to the metric $g$ !) Let $\rho_{1}(y)$ be a smooth function which is identically 1 for $|y|<1$, identically 0 for $|y|>2$ and satisfies $0 \leqq \rho_{1} \leqq 1$ in between. (Without loss of generality we may assume that the range of $y$ is at least $|y|<3$.) Set $\rho_{0}=1-\rho_{1}$. Define a new metric $h=\rho_{1}\left(d x^{2}+d y^{2}\right)+\rho_{0} g$. Then clearly $h$ satisfies the hypothesis of Theorem 2.

Since $g$ and $h$ are equal outside of a relatively compact neighborhood of $C$, there exist positive constants $k_{1}, k_{2}$ such that $k_{1} g^{i j} \leqq h^{l j} \leqq k_{2} g^{l j}$. Then one easily sees that for all smooth sections $\psi$ :

$$
\left(\frac{k_{1}}{k_{2}}\right)^{2} \frac{\int|D \psi|_{g}^{2} \sqrt{g} d^{2} x}{\int|\psi|_{g}^{2} \sqrt{g} d^{2} x} \leqq \frac{\int|D \psi|_{h}^{2} \sqrt{h} d^{2} x}{\int|\psi|_{h}^{2} \sqrt{h} d^{2} x} \leqq\left(\frac{k_{2}}{k_{1}}\right)^{2} \frac{\int|D \psi|_{g}^{2} \sqrt{g} d^{2} x}{\int|\psi|_{g}^{2} \sqrt{g} d^{2} x} .
$$

It follows immediately from the characterization of the first eigenvalue as the minimum of these quotients that

$$
k E_{1}(g) \leqq E_{1}(h) \leqq K E_{1}(g),
$$

where $k=\left(\frac{k_{1}}{k_{2}}\right)^{2}, K=\left(\frac{k_{2}}{k_{1}}\right)^{2}$ and the notation $E_{i}(g), E_{i}(h)$ has the obvious meaning. A few moment's reflection shows that by using the min-max characterization of the $j^{\text {th }}$ eigenvalues that the same is true for all discrete eigenvalues:

$$
k E_{j}(g) \leqq E_{J}(h) \leqq K E_{j}(g) .
$$

The bound of Theorem 1 now follows directly from that of Theorem 2 .

\section{Appendix}

Here we prove Theorem 3 and thus the bound Eq. (1). They follow immediately from the following theorem together with the fact that the curvature of $L^{\lambda}$ is $\lambda$ times that of $L$.

Theorem 4. Let $\phi$ be a section of $L \rightarrow M$. If $M$ has boundary suppose that $\phi$ is zero on its boundary and if $M$ is noncompact suppose that $\phi$ is square integrable. Then

$$
\left\|D_{A} \phi\right\|_{M}^{2} \geqq\left.\left|\int_{M} B\right| \phi\right|^{2} d^{2} x \mid .
$$

Equality holds if and only if the section $\phi$ is holomorphic or anti-holomorphic on each component of $M$.

Remark. Theorem 3 also follows immediately from the Weitzenbock type identity of Bochner-Kodaira-Nakano. See Demailly [8] and references therein.

We explain what we mean by holomorphic and anti-holomorphic. $M$ admits coordinates $(x, y)$, often called isothermal coordinates, with the property that in these coordinates the metric has the form $d^{2} s=\mu(x, y)^{2}\left(d x^{2}+d y^{2}\right)$ and $d x \wedge d y$ agrees with the orientation of $M$. Then $z=x+i y$ defines a complex coordinate on $M$. Set

$$
D_{z} \phi=\frac{1}{2}\left(D_{1} \phi-i D_{2} \phi\right)
$$


and

$$
D_{\bar{z}} \phi=\frac{1}{2}\left(D_{1} \phi+i D_{2} \phi\right),
$$

where the index 1 refers to $x$ and 2 to $y$. Then $\phi$ is holomorphic if $D_{\bar{z}} \phi=0$ and anti-holomorphic if $D_{z} \phi=0$.

Set $d z=d x+i d y$ and $d \bar{z}=d x-i d y$. Then we can form the combination

$$
\bar{\partial}_{A} \phi=D_{\bar{z}} \phi d \bar{z}
$$

which yields a well defined operator $\bar{\partial}_{A}: \Gamma(L) \rightarrow \Gamma\left(T^{(0,1)} \otimes L\right)$ over the whole manifold. $\left(T^{(0,1)}\right.$ denotes the complex line bundle with local sections $d \bar{z}$.) Similarly we can define $\partial_{A}: \Gamma(L) \rightarrow \Gamma\left(T^{(1,0)} \otimes L\right)$. One easily calculates that

$$
D_{A}=\bar{\partial}_{A}+\partial_{A}
$$

Sign Conventions. In this appendix we use the complex manifolds convention $D_{A} \phi=d \phi+i A \phi$ when $A$ is the connection form, as opposed to the physics convention, $D_{A} \phi=d \phi-i A \phi$, used in the rest of this paper. The reason for making this change of convention is so that the next theorem corresponds to those found in standard references on complex manifolds [4] and line bundles. With this convention $D_{1} D_{2} \phi-D_{2} D_{1} \phi=i F_{12} \phi$. The sign convention on the Hermitian inner product on $L$ is $(\phi, z \psi)=\bar{z}(\phi, \psi)$.

The above theorem is now an immediate consequence of the following

Theorem 5 (of Weitzenböck-Bochner type).

$$
\begin{aligned}
& 2\left\|\bar{\partial}_{A} \phi\right\|_{M}^{2}=\left\|D_{A} \phi\right\|_{M}^{2}-\int_{M} B|\phi|^{2}, \\
& 2\left\|\partial_{A} \phi\right\|_{M}^{2}=\left\|D_{A} \phi\right\|_{M}^{2}+\int_{M} B|\phi|^{2} .
\end{aligned}
$$

Proof. $\left\|\bar{\partial}_{A} \phi\right\|_{M}^{2}$ is obtained by integrating

$$
\begin{aligned}
\left(\bar{\partial}_{A} \phi, \bar{\partial}_{A} \phi\right) & =\frac{1}{4}\left(D_{1} \phi-i D_{2} \phi, D_{1} \phi-i D_{2} \phi\right)|d z|^{2} \\
& =\frac{1}{4}\left\{\left|D_{1} \phi\right|^{2}+\left|D_{2} \phi\right|^{2}+i\left[\left(D_{1} \phi, D_{2} \phi\right)-\left(D_{2} \phi, D_{1} \phi\right)\right]\right\} \frac{2}{\mu^{2}}
\end{aligned}
$$

Since $d^{2} x=\mu^{2} d x \wedge d y$ this integral is

$$
\left\|\bar{\partial}_{A} \phi\right\|_{M}^{2}=\frac{1}{2}\left[\left\|D_{A} \phi\right\|_{M}^{2}+i \int_{M}\left[\left(D_{1} \phi, D_{2} \phi\right)-\left(D_{2} \phi, D_{1} \phi\right)\right] d x d y\right] .
$$

We integrate the second term by parts. Form

$$
\alpha=\left(\phi, D_{A} \phi\right)=\left(\phi, D_{1} \phi\right) d x+\left(\phi, D_{2} \phi\right) d y .
$$

Then

$$
\begin{aligned}
d \alpha & =\left\{\left(D_{1} \phi, D_{2} \phi\right)-\left(D_{2} \phi, D_{1} \phi\right)+\left(\phi,\left[D_{1} D_{2}-D_{2} D_{1}\right] \phi\right)\right\} d x \wedge d y \\
& =\left\{\left(D_{1} \phi, D_{2} \phi\right)-\left(D_{2} \phi, D_{1} \phi\right)+\left(\phi, i F_{12} \phi\right)\right\} d x \wedge d y .
\end{aligned}
$$


Now the Dirichlet boundary conditions imply that $\alpha=0$ on the boundary of $M$. So by Stokes' formula, and the definition of $B: F_{12} d x \wedge d y=B d^{2} x$, we have

$$
i \int_{M}\left[\left(D_{1} \phi, D_{2} \phi\right)-\left(D_{2} \phi, D_{1} \phi\right)\right] d x \wedge d y=-\int_{M} B|\phi|^{2} d^{2} x .
$$

The first formula follows.

The second one is derived in exactly the same way.

QED.

The theorem has several interesting corollaries which appear to be well-known to the complex geometry community but which we have not found in the Schrödinger operator literature. In the complex geometry literature, bundles for which $B>0$ are called positive line bundles.

Corollary 1. Suppose that $M$ is compact without boundary and $B$ is constant. Then the ground state energy for the covariant Laplacian is $\frac{\left|c_{1}\right|}{\text { Area }(M)}$, where $c_{1}$ is the first Chern class of the bundle. In particular, if we take L to have Chern class 1 then the energy corresponding to $H(\lambda)$ is $\frac{|\lambda|}{\operatorname{Area}(M)}$.

Corollary 2. Suppose $M$ is compact without boundary and $B \geqq 0$. Let $c_{1}$ be the first Chern number of $L$, and $g$ be the genus of $M$. Then the dimension $m_{1}$ of the lowest eigenspace satisfies $m_{1} \geqq c_{1}-g+1$. If we take $L$ to have Chern class 1 , then the ground state multiplicity, $m_{1}(\lambda)$, for the covariant Laplacian $H(\lambda)$ on $L^{\lambda}$ satisfies

$$
m_{1}(\lambda)=\lambda-g+1
$$

for all $\lambda$ with $\lambda>2 g-1$.

Proof. From the First Bochner formula the lowest eigensections are in one-to-one correspondence with holomorphic sections. The theorem is then a direct translation of the Riemann-Roch theorem.

Acknowledgement. The author is happy to be able to acknowledge beneficial conversations with Cliff Taubes, Viktor Ginsburg, Andre Todorov, André Bellaiche, José Escobar, and Barry Simon. He especially would like to thank Cesar Castilho, supported by $\mathrm{CNP}_{\mathrm{q}}, \mathrm{Brazil}$, for performing the numerical analysis estimating $E_{*}$. This work was supported in part by NSF grant DMS-9122708 and a University of California Santa Cruz faculty research grant.

\section{References}

1. Bismut, J-M.: Large Deviations and the Malliavin Calculus. Basel-Boston: Birkhäuser, 1984

2. Berline, N., Getzler, E., Vergne, M.: Heat Kernels and Dirac Operators, Berlin, Heidelberg, New York: Springer, 1992

3. Bryant, R., Hsu, L.: Rigidity of Integral Curves of Rank Two Distributions. Invent. Math. 114, 435-461 (1993)

4. Chern, S-S.: Complex Manifolds without Potential Theory. Berlin, Heidelberg, New York: Springer, 2nd ed., 1979

5. Copson, E.T.: Asymptotic Expansions Cambridge Tracts in Mathematics and Mathematical Physics, Cambridge. Cambridge Univ. Press, 1967

6. Cycon, H., Froese, R., Kirsch, W., Simon, B.: Schrödinger Operators. Texts and monographs in physics, Berlin, Heidelberg, New York: Springer, 1987 
7. Demailly, J.P.: Champs magnétique et inégalités de Morse pur la d"-cohomologie. C.R.A.S., ser. I, 301, no. 4, 119-122 (1985)

8. Demailly, J.P.: Champs magnétique et inégalités de Morse pur la d"-cohomologie. Ann. Inst Fourier, 35 fasc. 4, (1985)

9. Eastham, M.S.P.: The Asymptotic solution of Linear Differential Systems. Oxford: Oxford Science Pub., 1989

10. Erdelyi, A.: Asymptotic Expansions. New York, London, Dover, 1956

11. Gromov, M.: Carnot-Caratheodory spaces seen from within. IHES preprint M/94/6, (221 pages), 1994

12. Guillemin, V., Uribe, A.: The Trace Formula for Vector Bundles. Bulletin of the A.M.S. 15 no. 2, 222-224 (1986)

13. Guillemin, V., Uribe, A.: Some Spectral Properties of Periodic Potentials. In: Lect. Notes in Math., No. 1256, Berlin, Heidelberg, New York: Springer, 1987, pp. 192-214

14. Guillemin, V., Uribe, A.: The Laplace Operator on the $n^{\text {th }}$ Tensor Power of a Line Bundle: Eigenvalues Which are Uniformly Bounded in $n$. Asymptotic Analysis, 1, 105-113 (1988)

15. Helffer, B.: Conditions nécessaires d'hypoellipticité. J. Diff. Eq. 44, 460-481 (1982)

16. Helffer, B.: Survey on linear PDE on Nilpotent Groups. In: Lect. Notes Math. 1077, Berlin, Heidelberg, New York: Springer, 1984, 210-252, esp. pp. 219-223

17. Helffer, B., Nourrigat, J.: Hypoellipticité Maximale pour des Opérateurs Polynomes de Champs de Vecteurs. Boston: Birkhäuser, 1985

18. Helffer, B.: Semi-classical analysis for the Schrödinger Operator and Applications. Lect. Notes in Math 1336, Berlin, Heidelberg, New York: Springer, 1980

19. Hòrmander, L.: Hypoelliptic Second Order Differential Equations. Acta. Math. 119, 147-171 (1967)

20. Landau, L.D., Lifshitz, E.M.: Quantum Mechanics, v. 3 Course of Theoretical Physics, Reading, MA, USA, Pergamom Press, 1958

21. Liouville, J.: Sur le développment des fonctions ou parties de fonctions en séries... . J. Math. Pure Appl. 2, 16-35 (1837)

22. Liu, W-S., Sussmann, H.J.: Shortest paths for subRiemannian metrics on rank two distributions. Preprint, to appear, Trans. A.M.S., 1994

23. Martinet, J.: Sur les Singularites Des Formes Differentialles. Ana. Insp. (Grenoble), vol. 20, no. 1, 95-198 (1970)

24. Mitchell, J.: On Carnot-Caratheodory Metrics. J. Diff. Geom. v. 21, 34-45 (1985)

25. Montgomery, R.: Abnormal Minimizers. To appear, SIAM J. Control and Opt., 1994

26. Rothschild, L.P., Stein, E.M.: Hypoelliptic differential operators and nilpotent groups. Acta Math. 137, 248-315 (1977)

27. Simon, B.: Semiclassical Analysis of Low Lying Eigenvalues I. Non-degenerate Minima: Asymptotic Expansions. Ann. Inst. Henri Poincaré, Physique Théorique 38, np. 3, 295-307 (1983)

28. Strichartz, R.: Sub-Riemannian Geometry. J. Diff. Geom. 24, 221-263 (1983)

29. Treves, F.: Analytic hypoellipticity of a class of pseudodifferential operators with double characteristics and applications to the $\bar{\partial}$-Neumann problem. Comm. P.D.E. 3, 475-642, esp. p. 478 and 501 (1978)

30. Vershik, A.M., Ya Gerhskovich, V.: Nonholonomic Dynamical Systems, Geometry of Distributions and Variational Problems. In: Dynamical Systems VII, ed. V.I. Arnol'd and S.P. Novikov, vol. 16 of the Encyclopaedia of Mathematical Sciences series, NY: Springer, 1994 (Russian original 1987) 
